\title{
Open access gastroscopy: second survey of current practice in the United Kingdom
}

\author{
J G Silcock, M G Bramble
}

\begin{abstract}
In June 1990 a survey of members of the endoscopy section of the British Society of Gastroenterology showed that $47 \%$ of respondents were offering some form of open access gastroscopy (OAG). Only 10\% offered true (non-censored) OAG. The survey was repeated in June 1994. The overall provision of OAG had risen to $74 \%$, most of whom were offering true OAG. Censored OAG is still widely practised and characterised by referral letters to a consultant in contrast with the use of referral forms $(p<0 \cdot 001)$. Referral forms are being increasingly used and are an effective way of capturing important data such as the patients' symptoms $(\mathbf{1 0 0} \%)$, previous treatment $(87 \%)$, non-steroidal anti-inflammatory drug or aspirin use $(78 \%)$, suspected diagnosis $(74 \%)$, and other medical conditions ( $72 \%$ ). Forms were used to establish clinical responsibility with the general practitioner in $64 \%$ of units. Standardised referral and reporting forms were used by $27 \%$ of respondents. A perceived inability to cope with the expected workload was still the most commonly cited reason for not being able to offer OAG. Although $20 \%$ of units with a single handed endoscopist were able to offer OAG, this compared with $68 \%$ of units with two or more endoscopists $(p<0 \cdot 001)$. Only three units indicated that an OAG service had had to be withdrawn, but a further 12 consultants (nine units) were now offering an age restricted service because of excessive workload. Two thirds of the respondents not offering OAG were hoping to do so in the near future. True OAG has increased from $10 \%$ to $41 \%$ in four years. (Gut 1997; 40: 192-195)
\end{abstract}

Keywords: open access gastroscopy.

In June 1990 a survey of 450 members of the endoscopy section of the British Society of Gastroenterology showed that $47 \%$ offered some form of open access gastroscopy (OAG), most censoring letters for 'clinical appropriateness'. Only $10 \%$ of respondents allowed unrestricted general practitioner access to gastroscopy services. ${ }^{1}$ In many cases it was unclear as to whether censoring was to keep numbers down or speed up the investigation of patients with a clear upper gastrointestinal history. It was also unclear how many gastroenterologists took on the responsibility of further investigation or treatment of the patient, or both, irrespective of the general practitioners' wishes.

The aim of this survey was to re-examine current practice after a further four years of experience and investigate the differences that exist between centres in terms of referral practice. In particular the questionnaire was carefully worded to tease out the differences between true and censored OAG.

\section{Definitions}

The definition of open access endoscopy was given as follows. 'The provision of a diagnostic endoscopic procedure by direct request of a general practitioner without prior hospital consultation, but including the provision of screening the appropriateness of any referral' (Fig 1).

If the normal procedure was for a general practitioner to request OAG by referral to a specific consultant (whether by letter or form) this was considered to be a censored request as the named consultant would approve (or not) that request and by definition be responsible for the investigation (even if not carried out personally). Requests for OAG sent to departments and not naming consultants were considered to be truly open access irrespective of whether a letter or form was used. Such requests would still need to be 'screened' by staff to ensure that the examination could be carried out safely, but this would not necessarily be a consultant.

\section{Methods}

In June 1994 the endoscopy section of the British Society of Gastroenterology had over 600 members, many of whom were living abroad or not practising in the National Health Service. A total of 465 questionnaires were posted to United Kingdom members with an explanatory letter. A single reminder was sent to non-responders after six weeks. The questionnaires were carefully worded to establish which of the various referral methods were in use. At the end of four months all returned questionnaires were analysed and differences examined, where appropriate, using the $\chi^{2}$ test.

Open access endoscopy refers to the provision of a diagnostic endoscopic procedure by direct request of general practitioners without prior hospital consultation but including the provision of 'screening' the appropriateness of any referral.

Figure 1: Definition of open access endoscopy. 
The responses are presented by individual consultant rather than by endoscopy unit as in some units consultants were split in terms of whether they offered OAG or not. This results in some skewing of the figures in favour of units offering OAG but does not affect the differences between true and censored referral patterns. This is also the way results were presented in the first survey.

\section{Results}

A total of 333 questionnaires were returned from individual members representing a response rate of $72 \%$. Twenty replies were returned as not relevant to the individual concerned. Figure 2 shows the age and specialty of respondents.

\section{OPEN ACCESS GASTROSCOPY}

This service was offered by 232 consultants $(70 \%)$, of whom 95 were performing OAG on receipt of a letter or form addressed to a consultant (censored) while 137 replies indicated that the examination was carried out following a referral letter or form to the department concerned. Thus $59 \%$ were offering true OAG.

Of the 232 replies, a slight majority were dependent on a letter from the general practitioner as the mode of referral (127-55\%), and were predominantly censored (that is, sent to a named consultant, 90 replies). Standardised referral forms were used by 109 respondents of which $84 \%$ (92 replies) were sent to departments (true OAG). A clear difference was seen between the two groups. True OAG was largley based on the receipt of a form while most letters were sent to consultants and therefore censored $\left(\chi^{2}=70 \cdot 08, \mathrm{p}<0 \cdot 001\right)$. Ten consultants offered both true and censored OAG, six by letter and four by both letter and form.

\section{Quality of information}

No attempt was made to assess the quality of information in referral letters as this was likely to vary enormously. However a structured request form is able to standardise information

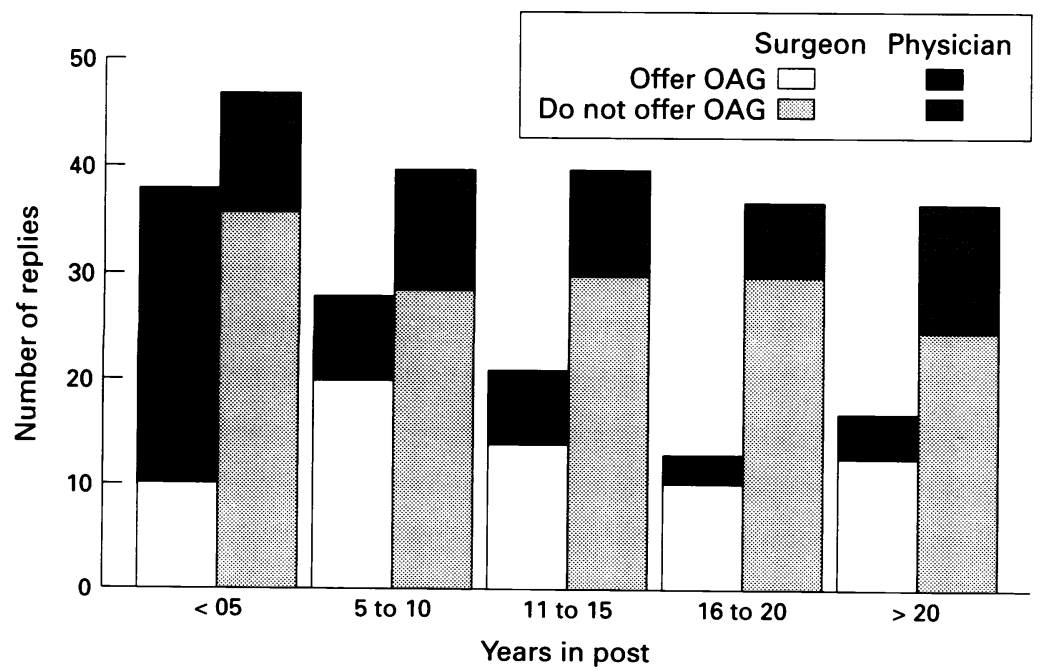

Figure 2: Age and specialty of respondents. to the clinician and the use of forms was characterised by a record of the patients' symptoms $(100 \%)$, previous treatment $(87 \%)$, non-steroidal anti-inflammatory drug or aspirin ingestion (78\%), suspected diagnosis $(74 \%)$, and the presence of other medical conditions $(72 \%)$.

\section{Guidelines}

Only $22 \%$ (51 replies) of consultants offering OAG had set out guidelines regarding the suitability of OAG in certain patients, for example, with dysphagia or anaemia. An age related policy was operated by $10 \%$ of consultants (not gastroscoping patients under 40 or 45 years of age) half of whom had previously been offering an unrestricted service.

In most units a pre-endoscopy health check was carried out by the nursing staff, although 66 replies $(28 \%)$ indicated that a doctor alone was responsible.

\section{Informing the general practitioner}

Two thirds of respondents indicated that results were returned to the general practitioner in a standardised (often computer generated) form, while $31 \%$ (73 replies) used a letter. Units accepting referrals by form were more likely to return the result in the same format compared with units accepting letters $\left(\chi^{2}=4.01, \mathrm{p}<0.05\right)$. Responsibility for clinical management was stated as remaining with the general practitioner on the referral form or in guidelines by $64 \%$ of respondents (149 replies).

\section{Willing or cajoled?}

Most consultants (75\%, 174 replies) stated that the service was initiated from hospital (Table I). Pressure from general practitioners was the only stated reason in $7 \%$ (16 replies) while 3\% (eight replies) indicated that the reason for OAG was managerial. Because some units had been offering OAG for a long time (Fig 3), 9\% of respondents (20 replies) did not know the reasons for establishing the service. In some cases $(5 \%, 12$ replies) the reasons were multifactorial. Most consultants felt that fundholding had made no difference to the number of requests for gastroscopy although $28 \%$ thought it had increased demand while 3\% (six replies) thought it had decreased referrals.

NO OPEN ACCESS GASTROSCOPY A total of 101 replies (30\%) indicated that they were not offering OAG. Reasons are shown in

TABLE I The initiator of the open access service $(n=232)$

\begin{tabular}{lcc}
\hline Initiator & Number & Percentage \\
\hline Consultant & 174 & 75 \\
Manager & 8 & 3 \\
General practitioner & 16 & 7 \\
Multifactorial & 12 & 5 \\
Not known & 20 & 9 \\
No reply & 2 & 1 \\
\hline
\end{tabular}


TABLE II Reasons for not offering OAG service $(n=101)$

\begin{tabular}{ll}
\hline Reasons & Percentage \\
\hline GPs will abuse the service & 28 \\
The unit would be unable to cope with the numbers & 73 \\
It offers no benefit to the patient & 21 \\
Disagree with the principle of open access & 21 \\
\hline
\end{tabular}

Table II. Almost two thirds of the units not offering OAG were hoping to do so in the near future $(65 \%, 65$ replies).

REDUCTION OR WITHDRAWAL OF OPEN ACCESS GASTROSCOPY

Three consultants (all different units) had stopped offering OAG since 1990 due to excessive workload. A further 12 consultants (nine units) now offered a restricted service (age related policy). The three units had only two, three, and four endoscopists respectively. Only $20 \%$ of units with a lone endoscopist are able to offer OAG (six of 30 replies) but this figure increases to $73 \%$ ( 220 of 303 replies) if two or more endoscopists are available $\left(\chi^{2}=32 \cdot 272, \mathrm{p}<0 \cdot 001\right)$.

OPEN ACCESS FLEXIBLE SIGMOIDOSCOPY The proportion of consultants offering OAG who also offer open access flexible sigmoidoscopy was $28 \%$ (66 replies). Most of these had been offering OAG for less than five years $(68 \%, 45$ replies).

\section{Discussion}

The purpose of this questionnaire was not to discover why consultants were offering OAG or even to determine the merits of one system versus another. The primary aim was to determine current practice four years on from a previous survey and after a time of great change in the National Health Service. Such change has to be managed and we had become aware that in many units the mechanics of offering OAG were often a mixture of old and new practices, leaving such issues as who was reponsible for the patients future management unclear.
The response to this survey was good, although slightly down on the results of the 1990 survey $(72 \% v 78 \%)$. It is not comprehensive because it only establishes the working practices of endoscopy section members. Nevertheless, most endoscopy units will have at least one consultant who is a member of the British Society of Gastroenterology and the endoscopy section encompasses the interests of those most likely to be working 'at the coal face'. As such, the survey is of interest to all endoscopists.

The overall provision of OAG has increased substantially with a total of 232 members now offering this service (70\%) compared with 167 $(47 \%)$ in $1990 .{ }^{1}$ The overall incidence of true OAG has increased from $10 \%$ to $41 \%$ although the 1990 figure is less accurate due to the nature of the first questionnaire. Taking into account the limitations of the first survey the overall incidence of censored OAG has fallen slightly from $37 \%$ to $30 \%$. These units operate a consultant based referral system with letters, or less commonly forms, being sent to individual specialists. This system is akin to an outpatient referral and implies consultant responsibility for the patients' management over and above performing the gastroscopy. It is quite different from the arrangement that exists for barium meal requests, where the general practitioner would not expect the radiologist to arrange further investigation or treatment, or both. In many cases the endoscopist will not be a consultant but a clinical assistant or hospital practitioner. ${ }^{2}$ It is thus inappropriate to transfer responsibility for patients to the hospital in the open access setting. All OAG services augment conventional referral pathways and OAG should not be seen as a way of gaining rapid access to a consultant opinion. Patients requiring a consultant opinion should still be referred to a gastroenterology outpatient clinic.

The reasons for the increase in OAG are probably multifactorial depending upon local facilities and the strength of feeling among local general practitioners that they should have this service. The data suggest that most OAG is consultant initiated, but this must be interpreted with caution as the increase

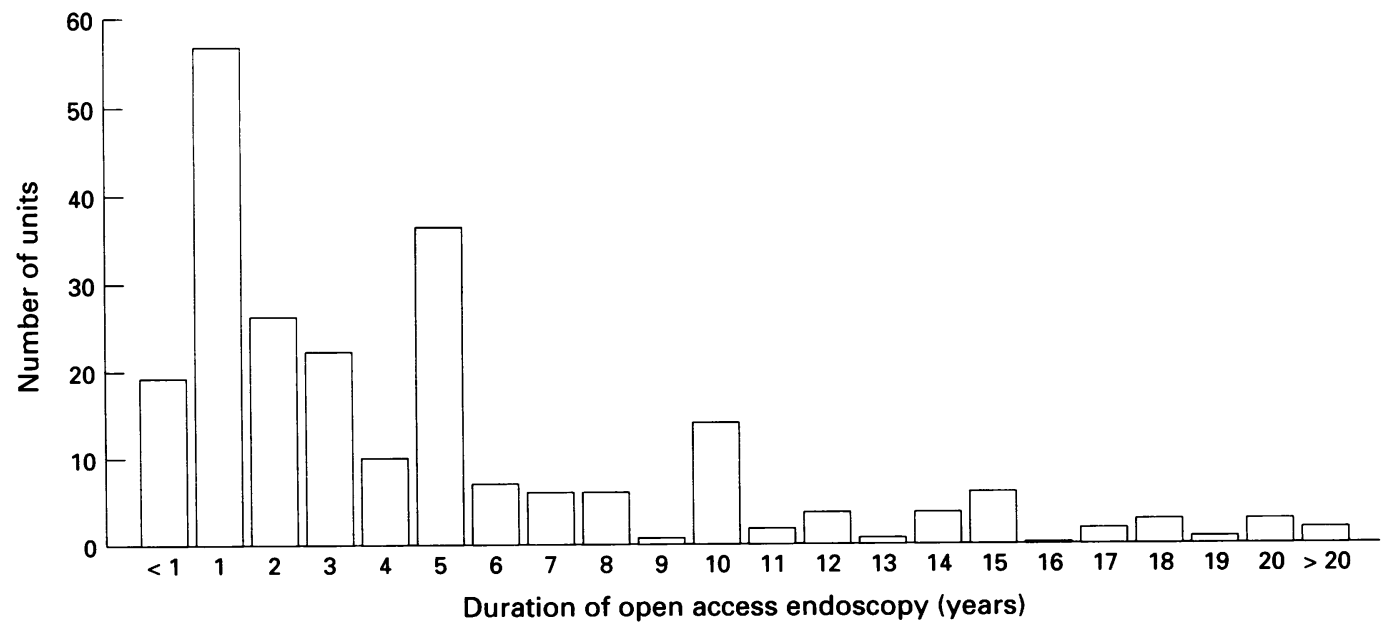

Figure 3: Histogram showing the increase in the number of units offering $O A G$. 
certainly coincides with the Health Service reforms and $15 \%$ admit that the pressure came from general practitioners or managers. Despite this, a significant minority of gastroenterologists $(30 \%)$ do not offer OAG, some of whom feel that it is not in the best interests of the patient. The pros and cons of OAG are debated elsewhere ${ }^{3-5}$ but it is important to note that a perceived inability to cope with numbers is still the most commonly cited reason for not offering this service. ${ }^{1}$ This survey should reassure those consultants that units that have established an OAG service have rarely had to withdraw this facility and only a small number of units have had to restrict OAG to patients over 40 or 45 years of age.

The number of endoscopists available to share the workload remains an important factor in the provision of OAG and this may be one area where a nurse endoscopist ${ }^{6}$ or subconsultant grade could make a significant impact in the development of OAG. It is unreasonable to expect a single handed gastroenterologist to provide such a service and this survey confirms that a solitary endoscopist is least likely to offer OAG. However the concerns that general practitioners might abuse such a service now seem unfounded. Apart from the data in this survey that show only isolated instances of overload, a recent survey from general practice confirms that referral rates, corrected for consultation rates and partnership equivalents, fall into a narrow band. ${ }^{7}$ Like many other areas in the health service demand continues to increase year on year and this can only be met by either restricting access or responding to the demand by changing practice. As the number of gastroenterologists is unlikely to increase sufficiently, training more endoscopists allows general practitioners to investigate patients quickly without a (sometimes unnecessary) outpatient appointment.

Although the availability of OAG is increasing, there are pronounced differences in the clinical practices reported between units. The use of forms seems to be an efficient way of reporting the patients' symptoms, concomitant medical conditions, use of medications, and the general practitioners' diagnosis. A standardised form can also be used to establish clear guidelines on clinical responsi- bility and give guidelines on the suitability of the service for certain patients. In this study the main difference between the use of forms and letters was that forms were usually sent to departments (analogous to a barium meal request) while letters were sent to a named consultant (analogous to an outpatient appointment request). We feel that this could lead to confusion as to who is looking after the patient and that 'best practice' should incorporate a system that uses a short but pertinent referral form sent to the endoscopy unit concerned with an equally clear report back to the general practitioner stating the diagnosis and (if necessary) brief advice. Follow up studies do suggest that subsequent treatment and management of the patient is appropriate in such circumstances. ${ }^{8}$

In conclusion, this survey shows that the provision of OAG is rapidly increasing, although not all safeguards are in place to establish clear lines of responsibility. In particular censored OAG remains a problem area where confusion might arise between the general practitioner and specialist. Although we believe that true $O A G$ is a safe and an effective use of resources in a climate of increasing demand, the British Society of Gastroenterology should now define 'best practice' for all endoscopy services. Current levels of referral often exceed the capacity of a gastroenterologist to see all patients requiring an endoscopy in the outpatient clinic. If the National Health Service is to be increasingly 'primary care led', then general practitioners must also participate in the establishment of national guidelines relating to best practice.

1 Bramble MG. Open access endoscopy: A nation-wide survey of current practice. Gut 1992; 33: 282-5.

2 Bramble MG, Cooke WM, Corbett WA, Cann P, Clarke D Contractor B, et al. Organising unrestricted open access gastroscopy in South Tees. Gut 1993; 34: 422-7.

3 Jones R Open access endoscopy. BMF 1985; 291 : 424-6.

4 Bramble MG. Open access endoscopy. Update Sept 1990; 395.

5 Heatley RV. Open access gastrointestinal endoscopy. Popular, but is it right? BMF 1993; 306: 1224 .

6 The Nurse Endoscopist. Report of the British Society of Gastroenterology Working Party, London: 1994.

7 Hungin AS, Bramble MG, O'Callaghan H. Reasons for the variations in the use of open access gastroscopy by general practitioners. Gut 1995; 36: 180-2.

8 Hungin AS, Thomas PR, Bramble MG, Corbett WA, Idle N, Contractor $\mathrm{B}$, et al. What happens to patients following open access gastroscopy? An outcome study from general practice. Br fु Gen Pract 1994; 44: 519-21. 